\title{
How Usual Is "Play As You Usually Would”? A Comparison of Naturalistic Mother-Infant Interactions with Videorecorded Play Sessions in Three Cultural Communities
}

\author{
Monika Abels, ${ }^{1}$ Zaira Papaligoura, ${ }^{2}$ Bettina Lamm, ${ }^{3}$ and Relindis D. Yovsi ${ }^{3}$ \\ ${ }^{1}$ Department of Communication and Information Sciences, Tilburg School of Humanities, Tilburg University, Tilburg, Netherlands \\ ${ }^{2}$ School of Psychology, Faculty of Philosophy, Aristotle University of Thessaloniki, Thessaloniki, Greece \\ ${ }^{3}$ Culture and Development, Institute of Psychology, University of Osnabrück, Osnabrück, Germany
}

Correspondence should be addressed to Monika Abels; mabels@uvt.nl

Received 17 March 2017; Accepted 27 June 2017; Published 12 September 2017

Academic Editor: Nobuo Masataka

Copyright (C) 2017 Monika Abels et al. This is an open access article distributed under the Creative Commons Attribution License, which permits unrestricted use, distribution, and reproduction in any medium, provided the original work is properly cited.

In developmental research, mothers are frequently asked to "play as you usually would." In this study, maternal behavior towards their three-month-olds in three cultural communities (Nso, Cameroon; Gujarati, India; Athens, Greece) was compared between videorecorded "play" situations and naturalistic observations. If there is consistency, videorecorded "play" episodes can be used as a proxy for daily behavior. Body contact, body stimulation, face-to-face situations, and object stimulation were coded. While individual mothers showed consistent levels of body contact and face-to-face and object stimulation in both situations, there were also high correlations across the different types of behaviors. Only body contact and object stimulation correlate significantly across behavioral frames but not with each other across or within either observational frame. They can therefore be understood as behaviors with some discriminatory power. Mothers generally show a higher frequency of behaviors in the videorecorded play situations than during the everyday observations across all three communities. However, the samples differ in the extent to which three of the four behaviors are seen more in the videorecorded play sessions. A broader and general understanding of mothers' ethnotheories and daily activities in each community is required in order to interpret videographed "play as you usually would" situations.

\section{Introduction}

A commonly used paradigm to study mother-infant interactions in developmental research has been to videotape "free play" situations of mothers with their infants. Mothers are frequently asked to "behave in their usual manner" [1, p. 868] or "as they normally would do" [2, p. 118]. These instructions are used to identify differences between mothers' interactional styles or infants' developmental outcomes. There are generally no explicit statements in the publications with respect to why a play situation is used. One reason could be that play is taken as a particularly relevant context for the child's development. While there are certain characteristics that differentiate play from other contexts (e.g., the lack of a specific goal), this also is usually not made explicit in publications. As these explanations are lacking, another reason to use the play instruction will be considered in this paper, namely, that mothers will show behaviors that are representative of their everyday behaviors.

Instructions to "play as you usually would" also have been used in cross-cultural research [3]. Using this instruction in research studies has led to interesting results on caregiverinfant interactions. The authors of these cross-cultural studies show concern for the potential inappropriateness of their procedures, focused on the appropriateness of their translations [4] or the procedure's ethnographic validity [5]. However, in many cultural communities playing with adults is not viewed as a valuable activity for adults or infants $[6,7]$. Play activities with babies, as understood from a Western point of view, tend to require exclusive attention to the child, which can keep mothers with heavy workloads from other essential tasks. Additionally, close-knit dyadic relationships, as can be forged in play interactions, are sometimes perceived as potentially harmful for the (extended) family and therefore discouraged 
[8]. Many caregivers focus primarily on negative signals of the infant and try to console them quickly and generally keep the infants calm rather than providing the active stimulation of play $[6,9]$. Hierarchy and respect socialization (cf. [10]) also are important for adult-child play: a senior person due to status or age does not play, even in an imagined coequal status, with a junior.

Previous research has shown that daily experiences of infants in different cultural communities differ vastly, e.g., [11]. Behavioral domains which differ widely across cultural communities include body contact, body stimulation, faceto-face interactions, and object play. Hewlett and Lamb [12] found that Aka infants are held app. 98\% of their waking time, Euro-American infants are held only $44 \%$ of their waking time. Body stimulation in the form of baby massage [13] or stimulation to accelerate motor development [14] is a daily practice in many parts of the world but not in Western societies. Face-to-face interactions between mother and child are common in places such as Italy or the USA but are far less common in India or Kenya [15]. Finally, although it seems universal that infants become interested in objects, whether or not adults make or provide specific toys for babies and whether there is specific tuition regarding object manipulation differ widely across cultures $[6,16]$. These four behavioral domains (body contact, body stimulation, face-to-face interactions, and object play) form the core of Keller's Component Model of Parenting, which is related to more pervasive cultural characteristics and developmental outcomes in the child (cf. [10]) and will be used as the basis for this study.

The age of the infants was chosen to be three months. At this age, infants have developed some new social skills such as the social smile [17] and a focus on their caregivers' faces [18]. Infants' motoric skills at this point are still limited. They generally have control over their head but cannot locomote, yet. Nevertheless, they are active partners when interacting with their caregivers [19].

Although concerns about play instructions have been voiced before, they have not been tested empirically as far as we know. The aim of this study is to test the relation between videorecorded play situations and naturalistic observations empirically for mothers from three cultural communities, namely, Gujarati villagers in India, Nso farmers in Cameroon, and families in Athens, Greece. Two of these communities (Gujarati and Nso) are rural, in developing economies, thus representing a large proportion of the human population. They share features such as being predominantly farmers and raising children to be part of a hierarchical social structure. However, they are located on different continents and differ in aspects such as religion, division of labor, and childcare arrangements. The third community (Athens) is urban and European and in many ways more comparable to the standard samples used in psychology. However, this sample is also characterized by a particular religion, high family involvement in child-care, and a traditionally patriarchal family system.

1.1. Research Questions/Hypotheses. If individual mothers behave consistently in naturalistic behaviors and "play as usual" events there should be a high correlation of the same behavior in the two types of situations. Other correlations should be similar to those found between behaviors within each frame (e.g., if body contact and body stimulation correlate highly in the observations, the same should be true in the videos and across the two observational frames).

Of course there are many reasons why a "play as usual" event would not be identical to the stream of everyday interaction. For example, mothers in every cultural community have tasks besides interacting with their infant and play as usual would be likely to involve more interactions. Therefore, an effect of the observational frame (naturalistic or defined play situations) can be expected showing a higher proportion of mother-child interactional behavior in the videorecorded play situation compared with the naturalistic daily routine.

Nonetheless, the results from both observational frames should show a cultural consistency. That is, the relative importance of the four very common mother-infant behaviors mentioned above (body contact, body stimulation, face-toface interactions, and object play) should remain the same across "play as usual" and naturalistic observational frames. This result would suggest that the "play as usual" instruction given to mothers does reflect what they usually do during the day when not asked to play. This would support the conclusion that play could be used as a proxy for cultural differences more broadly.

\section{The Three Communities}

2.1. Rural Gujarat, India. The families from rural Gujarat who participated in this study live in the Nandesari area of Vadodara taluka in Gujarat, India. The vast majority of the population of the Nandesari area is Hindu though there are also a few Muslim and Christian families. Families in the Nandesari area are either nuclear (about 64\%) or joint families (about 36\%) [20]. Even if a family lives as a nuclear family, there is usually very close contact with relatives living in the same compound or in neighboring houses [21].

Women are considered responsible for the household and for any cattle and livestock. Mothers of young infants rarely work in the fields and if they do, they generally do not take infants along. Infants are often asleep in cloth cradles in the house while the mother does her chores in and around the house. Often there are other potential caregivers such as grandparents present in the house or nearby.

The ideal for the socialization of children is the joint family system [22] that is preserved by patrilocal marriages and close relations between paternal relatives. Children are expected to show conformity, respect, and obedience to their parents [23] and loyalty is expected with the extended family. Too much attention should not be paid to the infant, because it would spoil the child [22]. Mothers additionally fear danger due to their inadvertent gaze-the evil eye [24] - and so the mother's love for her child is not for public display, to minimize envy of such a too-close tie [25], and emotionally charged interactions with the mother are minimized [26].

2.2. The Nso, Rural Cameroon. The Nso in this study live in Kikaikelaki in Bui division of the Northwest region of 
Cameroon. Rural Nso society is based in values and customs that stem from traditional animistic religion, often combined with Catholicism, Protestantism, and Islam [27]. Most households are monogamous apart from ranked persons with traditional titles, who are expected to be polygynous. There is close contact with relatives and neighbors living in the same lineage or family land.

Mothers return to working on their farms when the baby is about three months of age. Mothers are responsible for child-care but are helped by female relatives or elder siblings who take care of the child while the mother goes about her chores in the compound. They carry or hold the child as the mother prepares food, washes the dishes and clothes, gets water, and sweeps the compound.

Nso children are raised to be obedient, respect authority, and conform to the group. The child is highly valued as the reincarnation of a deceased ancestor and a gift from God. The attainment of the moral values of the society-such as social responsibility, commitment to harmony, and group stability-constitutes Nso-ness [28].

2.3. Athens, Greece. The Greek sample is from Athens, with more than 3 million inhabitants. The majority of the Greek population are Greek Orthodox Christians, though there are other Christian, Muslim, Jewish, and neopagan minorities.

The traditional Greek family is patriarchal in structure with the woman as the organizer of the household. However, women are now in the workforce in large numbers [29] and the patriarchal family structure has been eroding for a long time, particularly with highly educated [30] and urban families [31]. Still, there is a high involvement of grandparents in child-rearing (daily in approximately one-third of the families), with grandparents frequently providing practical, emotional, and financial support to nuclear families [32].

For Greeks, loyalty to family is of primary importance, taking precedence over other interests or personal needs [33]. Children are raised to show obedience and respect but also to attain educational achievements and uniqueness. The data in this paper were collected prior to the economic depression and political crisis in Greece.

\section{Method}

Forty-six three-month-old infants and their mothers were observed (Table 1). The samples differ significantly in terms of maternal age and education. These differences reflect the characteristics of the communities. Each infant was observed naturalistically 20 times for 15 minutes over a period of several days at different times of the day [8, 11]. Prior observational research showed that measurements stabilize when observing mother-infant dyads for app. 4-6 hours [34]. The same infants then also were videotaped for approximately 10 minutes with their mothers who were asked to "play with your child as you usually would." The videorecordings were generally carried out by the same researchers who previously did the observations. It is this contrast between the naturalistic and play frames for doing infantmother interaction observations that we compare in this study.
TABLE 1: Sample characteristics.

\begin{tabular}{|c|c|c|c|c|}
\hline$n$ & $\begin{array}{c}\text { Gujarat } \\
18\end{array}$ & $\begin{array}{c}\text { Nso } \\
20\end{array}$ & $\begin{array}{c}\text { Athens } \\
8\end{array}$ & \\
\hline Mothers' mean age & $22.1^{\mathrm{a}}$ & $24.3^{\mathrm{a}, \mathrm{I}}$ & $30.4^{\mathrm{b}, \mathrm{II}}$ & $* *$ \\
\hline $\begin{array}{l}\text { Mothers' formal } \\
\text { education (years) }\end{array}$ & $4.3^{\mathrm{a}, \mathrm{I}}$ & $7.5^{\mathrm{b}}$ & $10.0^{\mathrm{b}, \mathrm{II}}$ & $* * *$ \\
\hline Female children (\%) & 44 & 60 & $63^{\mathrm{I}}$ & n. s. \\
\hline Laterborn children (\%) & 67 & 60 & $50^{\mathrm{II}}$ & n. s. \\
\hline
\end{tabular}

Note. ${ }^{* *} p<.01{ }^{* * *} p<.001 ;{ }^{\text {a b }}$ homogeneous subsets. I: data from one participant missing. II: data from three participants missing.

Most of the data used in this study, except for the Greek observational data, have been used in previous publications $[4,8,11]$, though this is the first study to compare the frames. The Greek data collection was coordinated by the second author of this paper and the observations were made by graduate students. While it was necessary for the research team to retain personal information about the participants while the study was ongoing (e.g., name, address, telephone number), these data were not saved afterwards and all participants were assigned a code by which the data were identified.

3.1. Coding. Caregivers' behaviors in both observational frames were coded by trained observers according to the Component Model of Parenting [4, 10]. Time sampling procedures were used in both observational frames. In the naturalistic observations, ten seconds were observed, followed by 20 seconds to do the (paper and pen) coding. When coding the videos, the tape could be stopped and replayed so that the whole 10-minute period could be coded. The final scores used in the analyses were the percentages of intervals in which each behavior occurred. The four behavioral domains are not mutually exclusive.

Body contact included any contact between the mother's and the child's body. Body contact generally does not involve movements of the bodies against each other, which is coded as body stimulation. Contact with the mother's hands was generally coded in the body stimulation system, not as body contact. Body contact was coded when it occurred for at least five seconds of the 10-second interval.

Body stimulation included stimulation of the infant's whole body (e.g., rocking) and body parts (e.g., exercising arms and legs) and pinching, massaging, stroking, or rubbing the child with the hand or the face. Body stimulation generally involves movement or a posturally challenging position for the child. Categories were coded if they occurred within a 10 -second interval.

Face-to-face context was defined as the effort of a mother to position her body and head towards her infant in a way that allowed face-to-face interaction. Face-to-face interaction was coded when the mother created a face-to-face situation for at least five seconds of the 10 -second interval.

Object stimulation was defined as the maternal effort of attracting the attention of the infant to an object in a playful way or following the infants' attention towards an object. The object could be touched by her and/or the child but could 
TABLE 2: Correlations between mothers' behaviors in videorecorded play situations and naturalistic observations.

\begin{tabular}{|c|c|c|c|c|}
\hline & \multicolumn{4}{|c|}{ Videorecorded play } \\
\hline & Body contact & Body stimulation & Face-to-face & Object stimulation \\
\hline \multicolumn{5}{|c|}{ Naturalistic observations } \\
\hline Body contact & $.45^{* *}$ & $.54^{* * *}$ & $.40^{* *}$ & .20 \\
\hline Body stimulation & .15 & -.17 & $-.26^{+}$ & -.02 \\
\hline Face-to-face & .23 & $.68^{* * *}$ & $.64^{* * *}$ & $.32^{*}$ \\
\hline Object stimulation & -.17 & .24 & $.29^{+}$ & $.37^{*}$ \\
\hline
\end{tabular}

Note. $N=46{ }^{+} p<.1,{ }^{*} p<.05,{ }^{* *} p<.01$, and ${ }^{* * *} p<.001$ (2-tailed).

TABLE 3: Correlations between mothers' behaviors in videorecorded play situations and naturalistic observations.

\begin{tabular}{|c|c|c|c|c|}
\hline & Body contact & Body stimulation & Face-to-face & Object stimulation \\
\hline Body contact & & .13 & .13 & .01 \\
\hline Body stimulation & -.10 & & $.68^{* * *}$ & $.32^{*}$ \\
\hline Face-to-face & $.60^{* * *}$ & .05 & & $.37^{*}$ \\
\hline Object stimulation & -.01 & .15 & $.47^{* *}$ & \\
\hline
\end{tabular}

Note. $N=46 ;{ }^{*} p<.05,{ }^{* *} p<.01$, and ${ }^{* * *} p<.001$ (2-tailed); numbers in bold refer to correlations within the videorecorded play situation; numbers in italic refer to the naturalistic observation.

also be used visually/acoustically. The occurrence of object stimulation was coded when an object was used within a 10second interval.

For the videographed play interactions the reliabilities for body contact, body stimulation, object stimulation, and faceto-face context were calculated on the basis of a sample of 10 video sequences (from different cultural communities), analyzed by two different coders. To obtain a coefficient of agreement, Cohen's Kappa was calculated and resulted in $K=.86$ for body contact, $K=.90$ for body stimulation, $K=.99$ for object stimulation, and $K=.85$ for face-to-face interaction.

For the naturalistic observations, coders were trained with the help of videographed mother-infant interactions and daily routine situations from different cultural communities. We calculated Cohen's Kappa for the assessment of 8 different 20-minute tapes for each pair of coders. Coders were considered reliable as soon as they reached Kappa values for all coding categories above .70. As the training was done for a more detailed coding scheme $[8,11]$ which is collapsed to the four parenting domains in this paper, reliability can be expected to have been higher. Additionally, the first 5 home visits in rural Gujarat were done by pairs of coders to confirm interrater agreement during fieldwork. Kappa values remained above .70 for all coded categories [8]. Only one trained coder was present at the other sites so that later reliability checks could not be done there.

\section{Results}

To test the first hypothesis that individual mothers' behaviors should be consistent across play and naturalistic frames we correlated mothers' behaviors in the two observational frames with each other. The results are depicted in Table 2. As can be seen, there are significant correlations between body contact, face-to-face context, and object stimulation in the two observational frames. However, there are equally strong correlations with other behavioral systems, for example, between face-to-face context during the naturalistic observations and body stimulation in the videorecorded play situation. Body contact and objects stimulation present the clearest picture by correlating highly across frames but not with each other.

When compared to the correlations between different behaviors within the videorecorded play situations and the observations, some significant correlations emerge as well (cf. Table 3). However, there is only some convergence with the interframe correlations. Of the four significant correlations among the interframe correlations, only two are in line with the correlations within the videorecorded play situation and the naturalistic observations, respectively. Of the other 8 (nonsignificant or marginally significant) interframe correlations four were in line with the correlations found in the videos and six were in line with the correlations in the observations. Again body contact and object stimulation emerge as behaviors that are also unrelated within each of the observational frames.

To test the influence of the observational frame and the cultural consistency across the frames, we calculated repeated measures MANOVA with the observational frame (naturalistic observation versus playing video) as the repeated measures factor, the mothers' cultural community as an independent variable, and the behavior types (body contact, body stimulation, face-to-face context, and object stimulation) as dependent variables. The analysis shows an effect for observational frame for all types of behaviors, with the proportion of behaviors in the videorecorded play situation being higher than in the observations $(F(4,40)=115.93$, partial eta squared $=.92$, and $p<.001$; body contact: $F(1,43)=51.61$, partial eta squared $=.55$, and $p<.001$; body stimulation: $F(1,43)=215.12$, partial eta squared $=$ .83 , and $p<.001$; face-to-face interaction: $F(1,43)=143.80$, partial eta squared $=.77$, and $p=.001$; object stimulation: $F(1,43)=14.15$, partial eta squared $=.25$, and $p<.001)$. 


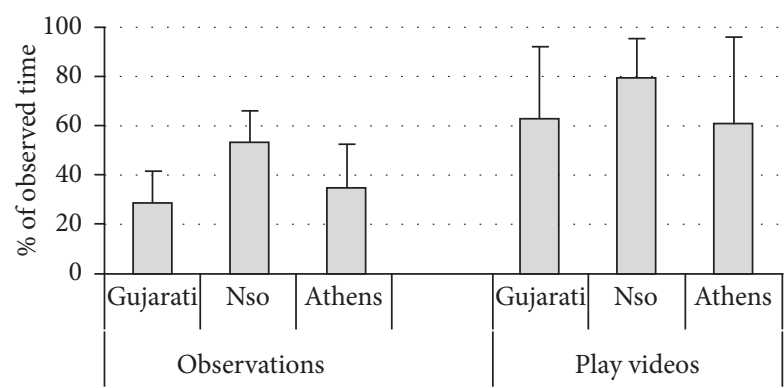

(a) Body contact: no significant interaction effect

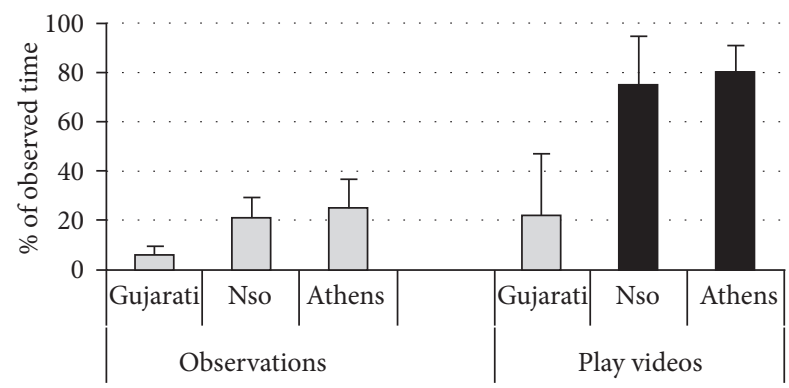

(c) Face-to-face interaction

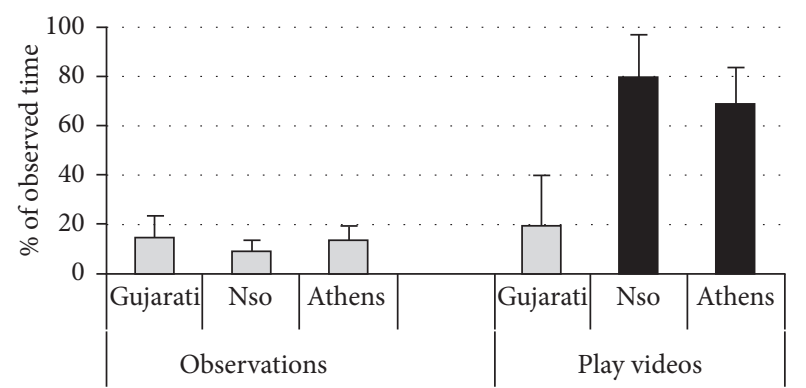

(b) Body stimulation

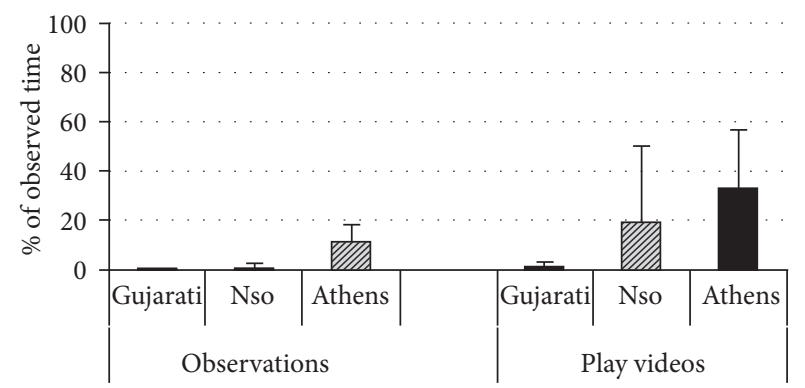

(d) Object stimulation

FIGURE 1: Observational frame $*$ cultural community interactions. Error bars represent one standard deviation and colors homogeneous subgroups.

If this higher proportion of behavioral types in the videorecorded play situations was similar across cultural communities, there should be no interaction effects between cultural community and observational frame. However, there are interaction effects on three of the four behavioral domains, the exception being body contact $(F(8,82)=7.25$, partial eta squared $=.41$, and $p<.001$; body contact: $F(2,43)=0.62$, partial eta squared $=.03$, and $p=.541$; body stimulation: $F(2,43)=61.60$, partial eta squared $=.74$, and $p<.001$; face-to-face interaction: $F(2,43)=17.26$, partial eta squared $=.45$, and $p<.001$; object stimulation: $F(2,43)=3.64$, partial eta squared $=.15$, and $p=.035$ ). Post hoc comparisons (cf. Figures 1(a)-1(d)) reveal that while the Gujarati mothers behave very similarly in both observational frames, Nso and Athenian mothers show a much higher frequency of body stimulation and face-to-face behaviors in the videorecorded play situations than during observations.

There also are cultural differences in all four behavioral domains $(F(8,82)=11.04$, partial eta squared $=.52$, and $p<.001$; body contact: $F(2,43)=7.85$, partial eta squared $=$ .27 , and $p=.001$; body stimulation: $F(2,43)=34.94$, partial eta squared $=.62$, and $p<.001$; face-to-face interaction: $F(2,43)=50.81$, partial eta squared $=.70$, and $p<.001$; object stimulation: $F(2,43)=9.32$, partial eta squared $=.30$, and $p<.001)$.

\section{Discussion}

Mothers showed a higher frequency of behaviors in the videorecorded play situations than in the naturalistic observations. This was true for all four behavioral systems assessed in this study. This higher behavioral frequency in the play interactions was expected because mothers in all three cultural communities have other activities and chores in addition to playing with their infant. The instruction to play excludes most activities the mother would otherwise be doing while with the child.

When mothers are asked to play with their infants "as they usually would" they show behaviors that are only partially consistent with their behaviors towards their infants throughout the day. There are equally high correlation between unrelated behavioral domains such as body contact during the observations and face-to-face interaction in the play videos or face-to-face context during the observations and body stimulation in the play videos. Therefore, generally the behaviors produced in a videorecorded play session should not be interpreted as representative of the mothers' behavior throughout the day on an individual level. There may be some behaviors that have a predictive value. However, a detailed analysis of these behaviors is necessary before utilizing only "play as you usually would" videos in research is justified. According to the coding scheme used here, body contact and object stimulation may be candidates that produce more clear-cut predictions because they show significant correlation within the behavioral domains across observational frames but do not intercorrelate significantly with each other. These characteristics may also be the reason that it is precisely these behaviors that are predictive when used in longitudinal studies [35]. Further research is necessary to substantiate this result and establish connections of play behaviors with everyday behaviors for other behavioral domains, for example, sensitivity ratings [36].

The culture by observational frame interaction shows that an interpretation of the videorecorded play session as 
representative of the respective cultural community's style of interacting with infants is also problematic. While body contact is consistent across observational frames, Gujarati mothers hardly change their behaviors in the other domains when asked to play while Nso and Athenian mothers do. This may be due to the particular cultural community that the Gujarati mothers live in, in which dyadic interactions are somewhat discouraged and play is a more prominent feature of interactions between infants and nonmaternal caregivers [8]. Additionally, certain body stimulation behaviors-such as baby massage [21] or practicing a standing position during defecation-are shown by Gujarati caregivers but would be part of a bathing/personal hygiene routine rather than being considered as "play" and are therefore absent in the videos.

However, there may also be more general behavioral tendencies at work, which are related to culture-specific emphasis or attenuation of behaviors. A study on Nso and German mothers that compared videorecorded motherinfant play in two settings had similar results [37]. While Nso mothers produced more behaviors during play in a lab than in a home environment, the reverse was true for German mothers. What leads mothers in some communities to emphasize their behaviors while others do not would be an empirical question for the future.

Besides the instruction to play in this study there was also the potential influence of the recording method: while the observations were coded on the spot with paper and pencil, the play situations were videorecorded. While some researchers claim that the participants "forget" the presence of the camera, others have tried to overcome its influence by concealment or deception (cf. [38] for a discussion). We would argue that an observer with paper and pencil may be as intrusive as a researcher with a camera. This assumption is supported by previous results in which $80 \%$ of the observed parents reported at least some degree of influence of being observed [39]. This is a methodological question for further research. However, the methods were consistent across communities and naturalistic/play instructed interactions. It is highly unlikely that the use of video or direct observation would have affected the clear cross-cultural differences and the variations in interactions with or without play instructions.

In conclusion, the "play as you usually would" frame provides an insight into socially acceptable play behavior as defined by the particular cultural community. However, "we can easily imagine cultures where parent-child play is absent, but when parent-child play does occur it is $[\cdots]$ recognizably play" [40, p. 134]. In these cultures play may be produced by mothers only in special circumstances, for instance, if they are asked to play by a researcher. This instruction may intensify certain behaviors, which become interpretable when they are supported by a thorough understanding of the meaning of play for mothers from different cultural communities. However, to understand the mothers' influences on their infants' development it is necessary to observe mother-infant dyads in a variety of situations [34]. It has been noted that parent-infant play is rather an exception than a rule in human cultures that is brought about by certain characteristics [41]. Among these are that infants live in contexts in which other children are not easily available as play mates as in harsh climatic conditions or urban middleclass families in industrialized societies (cf. [41]). Being with the mother is also agedependent with other interactional partners becoming more important as infants grow older (e.g., for North Indian infants [15]).

Interestingly a similar pattern holds for our great ape relatives. For instance, mothers of several great ape species have been observed to play with their infants [42-46]. Chimpanzee mothers engage in some of the same behaviors, namely, body contact and eye gaze, as assessed here for human mothers [45] while mother-infant object play seems to be rare in chimpanzees and bonobos [47]. In chimpanzees, as in young human infants, mothers have a greater social input in the interaction [48] but mothers may react to infants attempts to suckle or move away by playing [42]. At later ages, play continues to have an important role in immature great apes' lives but generally contact with the mother declines [49] and she may become an unlikely choice as a play mate (cf. [50]).

Although on a personal level body contact and object play may be candidates for predictive behaviors from the "play as you usually would" frame, overall the data presented here show that behaviors displayed in this frame are not sufficient to understand caregivers' representative behavior towards infants. A combination of naturalistic observations and play as usual within each community would ideally be necessary, as would be interviews to understand the cultural models of both play and parenting more generally, which influence what a mother will do in each frame. Starting from those data an appropriately framed structured observation of babies and caretakers can be developed. Together they will provide a rich and valuable approach to cross-cultural and within-culture studies of parenting and early child development.

\section{Additional Points}

Information on funding and acknowledgments for this work can be found in $[8,11,51]$.

\section{Disclosure}

Most of the data in this paper has been used in other publications. However, the two observational frames have not been compared or used together in any of these previous publications.

\section{Conflicts of Interest}

The authors declare that there are no conflicts of interest regarding the publication of this paper.

\section{Acknowledgments}

The authors are grateful to Heidi Keller and the Culture and Development Department at the University of Osnabrück who coordinated the data collection and analysis. They would also like to thank Tom Weisner for his helpful comments on this paper. 


\section{References}

[1] M. H. Bornstein, C. S. Tamis-LeMonda, C.-S. Hahn, and O. M. Haynes, "Maternal responsiveness to young children at three ages: longitudinal analysis of a multidimensional, modular, and specific parenting construct," Developmental Psychology, vol. 44, no. 3, pp. 867-874, 2008.

[2] T. Hofer, A. Hohenberger, P. Hauf, and G. Aschersleben, "The link between maternal interaction style and infant action understanding," Infant Behavior and Development, vol. 31, no. 1, pp. 115-126, 2008.

[3] M. H. Bornstein, O. M. Haynes, L. Pascual, K. M. Painter, and C. Galperín, "Play in two societies: Pervasiveness of process, specificity of structure," Child Development, vol. 70, no. 2, pp. 317-331, 1999.

[4] H. Keller, A. Lohaus, P. Kuensemueller et al., "The bio-culture of parenting: evidence from five cultural communities," Parenting: Science and Practice, vol. 4, no. 1, pp. 25-50, 2004.

[5] L. R. Cote and M. H. Bornstein, "Child and mother play in three U.S. cultural groups: comparisons and associations," Journal of Family Psychology, vol. 23, no. 3, pp. 355-363, 2009.

[6] D. F. Lancy, "Accounting for variability in mother-child play," American Anthropologist, vol. 109, no. 2, pp. 273-284, 2007.

[7] S. Gaskins, W. Haight, and D. F. Lancy, "The cultural construction of play," in Play and Development: Evolutionary, Sociocultural and Functional Perpectives, A. Göncü and S. Gaskins, Eds., pp. 179-202, Lawrence Erlbaum, Hillsdale, NJ, USA, 2007.

[8] M. Abels, H. Keller, P. Mohite et al., "Early socialization contexts and social experiences of infants in rural and urban Gujarat, India," Journal of Cross-Cultural Psychology, vol. 36, no. 6, pp. 717-738, 2005.

[9] R. A. LeVine, S. Dixon, S. LeVine, and R. A. LeVine, Child Care and Culture: Lessons from Africa, Cambridge University Press, 1996.

[10] H. Keller, Cultures of Infancy, Lawrence Erlbaum Associates, Mahwah, NJ,USA; Psychology Press, 2007.

[11] H. Keller, M. Abels, B. Lamm, R. D. Yovsi, S. Voelker, and A. Lakhani, "Ecocultural effects on early infant care: A study in Cameroon, India, and Germany," Ethos, vol. 33, no. 4, pp. 512541, 2005.

[12] B. S. Hewlett and M. E. Lamb, "Integrating evolution, culture and developmental psychology: explaining caregiver-infant proximity and responsiveness in central Africa and the USA," in Between Culture and Biology, H. Keller, Y. H. Poortinga, and A. Schölmerich, Eds., p. 241, Cambridge University Press, 2002.

[13] M. E. Walsh Escarce, "A cross-cultural study of Nepalese neonatal behavior," in The Cultural Context of Infancy, Volume One: Biology, Culture, and Infant Development, J. K. Nugent, B. M. Lester, and T. B. Brazelton, Eds., pp. 65-86, Ablex Publishing Corporation, Norwood, NJ, USA, 1989.

[14] C. M. Super, "Environmental effects on motor development: the case of 'African infant precocity"' Developmental Medicine and Child Neurology, vol. 18, no. 5, pp. 561-567, 1976.

[15] D. Sharma and R. A. LeVine, "Child care in India: a comparative developmental view of infant social environments," New Directions for Child Development, no. 81, pp. 45-67, 1998.

[16] R. Bakeman, L. B. Adamson, M. Konner, and R. G. Barr, "!Kung infancy: the social context of object exploration," Child Development, vol. 61, no. 3, pp. 794-809, 1990.

[17] R. N. Emde and R. J. Harmon, "Endogenous and exogenous smiling systems in early infancy," Journal of the American Academy of Child Psychiatry, vol. 11, no. 2, pp. 177-200, 1972.
[18] H. Keller and G. Gauda, "Eye contact in the first months of life and its developmental consequences," in Psychobiology and Early Development, H. Rauh and H. C. Steinhausen, Eds., vol. 46 of Advances in Psychology, pp. 129-143, Elsevier, Amsterdam, Netherlands, 1987.

[19] C. Trevarthen, "Playing into reality: conversations with the infant communicator," in Winnicott Studies, vol. 7, pp. 67-84, 1993.

[20] A. Lakhani, S. Ganju, and P. Mahale, Reproductive and Child Health Status in the Nandesari Area, Deepak Charitable Trust, New Delhi, India, 1997.

[21] M. Abels, Baby Massage in Rural Gujarat, India. Physical Growth, Motor Development and Caregivers' Ethnotheories, University of Osnabrueck, 2002.

[22] J. L. Roopnarine, M. W. Lu, and M. Ahmeduzzaman, "Parental reports of early patterns of caregiving, play and discipline in India and Malaysia," Early Child Development and Care, vol. 50, no. 1, pp. 109-120, 1989.

[23] S. Kakar, The Inner World: A Psycho-Analytic Study of Childhood and Society in India, Oxford University Press, 1978.

[24] M. Abels, The Expression of Emotional Warmth: Ethnotheories of Rural and Urban Indian Mothers and Grandmothers, University of Osnabrueck, Osnabrück, Germany, 2007.

[25] N. Chaudhary, Listening to Culture, Sage, New Delhi, India, 2004.

[26] S. N. Kurtz, All the Mothers are One, Columbia University Press, New York, NY, USA, 1992.

[27] J. L. Banadzem, "Catholicism and Nso traditional beliefs," in African Crossroads: Intersections between History and Anthropology in Cameroon, I. Fowler and D. Zeitlyn, Eds., pp. 125-140, Berghahn Books, Providence, RI, USA, 1996.

[28] R. D. Yovsi, Ethnotheories about Breastfeeding and Mother-Infant Interaction, Lit Verlag, 2003.

[29] N. Kyriazis, "Women's employment and gender relations in Greece: Forces of modernization and tradition," European Urban and Regional Studies, vol. 5, no. 1, pp. 65-75, 1998.

[30] C. Safilios-Rothschild, "A comparison of power structure and marital satisfaction in Urban Greek and French families," Journal of Marriage and the Family, vol. 29, no. 2, pp. 345-352, 1967.

[31] J. Georgas, "Intrafamily acculturation of values in Greece," Journal of Cross-Cultural Psychology, vol. 22, no. 4, pp. 445-457, 1991.

[32] A. Svensson-Dianellou, P. K. Smith, and E. Mestheneos, "Family help by Greek grandparents," Journal of Intergenerational Relationships, vol. 8, no. 3, pp. 249-263, 2010.

[33] C. Coulacoglou and E. Kotsoni, "The application of the fairy tale test in Greece," in Exploring the Child's Personality Developmental, Clinical and Cross Cultural Applications of the Fairy Tale Test, C. Coulacoglou, Ed., pp. 243-263, Charles C Thomas Publisher, Springfield, Ill, USA, 2008.

[34] B. Leyendecker, M. E. Lamb, A. Schölmerich, and D. M. Fricke, "Contexts as moderators of observed interactions: a study of Costa Rican mothers and infants from differing socioeconomic backgrounds," International Journal of Behavioral Development, vol. 21, no. 1, pp. 15-34, 1997.

[35] H. Keller, R. Yovsi, J. Borke, J. Kärtner, H. Jensen, and Z. Papaligoura, "Developmental consequences of early parenting experiences: Self-recognition and self-regulation in three cultural communities," Child Development, vol. 75, no. 6, pp. 17451760, 2004. 
[36] M. D. S. Ainsworth, “Maternal Sensitivity Scales," 1969, http:// www.psychology.sunysb.edu/attachment/measures/content/ ainsworth_scales.html.

[37] B. Lamm, H. Gudi, C. Freitag et al., "Mother-infant interactions at home and in a laboratory setting: a comparative analysis in two cultural contexts," Journal of Cross-Cultural Psychology, vol. 45, no. 6, pp. 843-852, 2014.

[38] E. Connors and S. M. Glenn, "Methodological considerations in observing mother-infant interactions in natural settings," in Psychological Research: Innovative Methods and Strategies, J. Haworth, Ed., pp. 139-152, Routledge, London, UK, 1996.

[39] A. Russell, G. Russell, and D. Midwinter, "Observer influences on mothers and fathers: self-reported influence during a home observation," Merrill-Palmer Quarterly, vol. 38, no. 2, pp. 263283, 1992.

[40] K. MacDonald, "Parent-child play: an evolutionary perspective," in Parent-Child Play: Descriptions and Implications, $\mathrm{K}$. MacDonald, Ed., pp. 113-143, State University of New York Press, Albany, NY, USA, 1993.

[41] D. F. Lancy, The Anthropology of Childhood, Cambridge University Press, 2008.

[42] J. van Lawick Goodall, "Mother chimpanzees' play with their infants," in Play Its Role in Development and Evolution, J. S. Bruner, A. Jolly, and K. Sylva, Eds., pp. 262-267, Penguin Books, Harmondsworth, UK, 1976.

[43] T. Enomoto, "Social play and sexual behavior of the bonobo (Pan paniscus) with special reference to flexibility," Primates, vol. 31, no. 4, pp. 469-480, 1990.

[44] L. C. Miller and R. D. Nadler, "Mother-infant relations and infant development in captive chimpanzees and orang-utans," International Journal of Primatology, vol. 2, no. 3, pp. 247-261, 1981.

[45] K. A. Bard, "Evolutionary roots of intuitive parenting: Maternal competence in chimpanzees," Early Development and Parenting, vol. 3, no. 1, pp. 19-28, 1994.

[46] D. Fossey, "Development of the mountain gorilla (Gorilla gorilla beringei): the first thirty-six months," in The Great Apes, D. A. Hamburg and E. R. McCown, Eds., pp. 139-184, BenjaminCummings, Menlo Park, CA, USA, 1979.

[47] K. A. Bard and J. Vauclair, "The communicative context of object manipulation in ape and human adult-infant pairs," Journal of Human Evolution, vol. 13, no. 2, pp. 181-190, 1984.

[48] J. R. Horvat and H. C. Kraemer, "Infant socialization and maternal influence in chimpanzees," Folia Primatologica, vol. 36, no. 1-2, pp. 99-110, 1981.

[49] E. V. Lonsdorf, K. E. Anderson, M. A. Stanton et al., "Boys will be boys: sex differences in wild infant chimpanzee social interactions," Animal Behaviour, vol. 88, pp. 79-83, 2014.

[50] K. P. Lewis, "Social play in the great apes," in The Nature of Play: Great Apes and Humans, A. D. Pellegrini and P. K. Smith, Eds., pp. 27-53, Guilford Press, 2005.

[51] H. Keller, Z. Papaligoura, P. Kuensemueller et al., "Concepts of mother-infant interaction in Greece and Germany," Journal of Cross-Cultural Psychology, vol. 34, no. 6, pp. 677-689, 2003. 


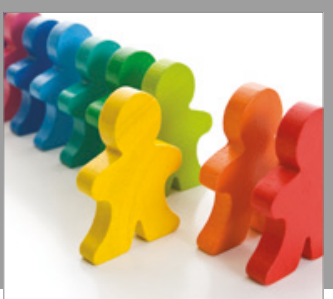

Autism

Research and Treatment
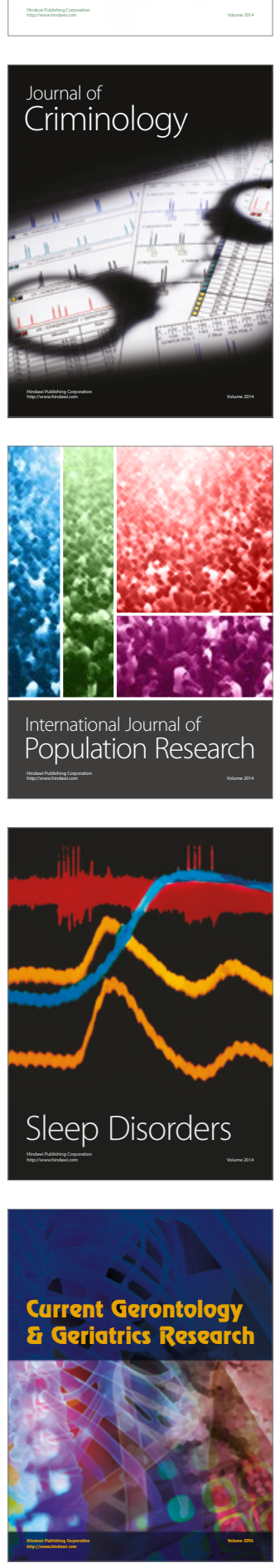

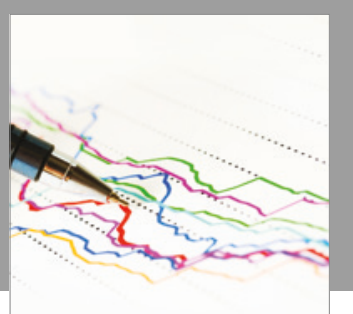

Economics

Research International

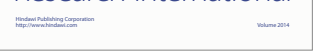

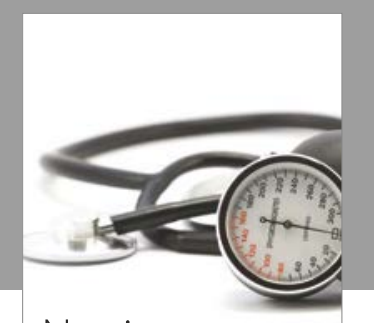

Nursing

Research and Practice

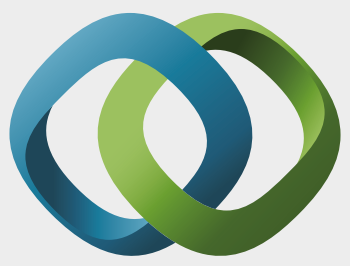

\section{Hindawi}

Submit your manuscripts at

https://www.hindawi.com
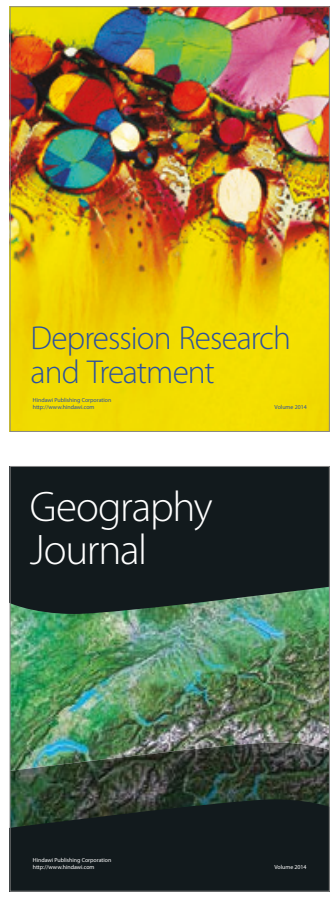
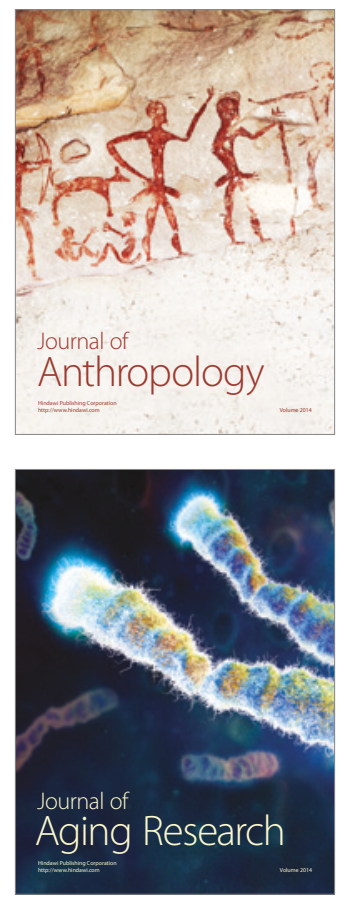
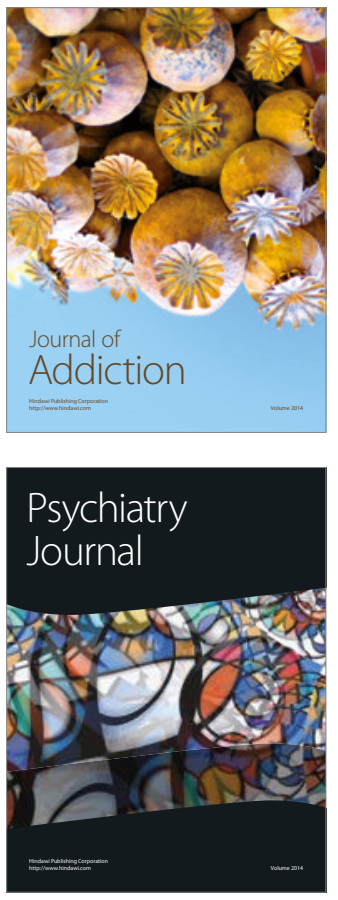

Child Development

Research

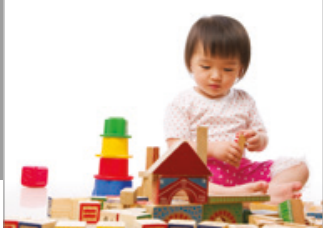

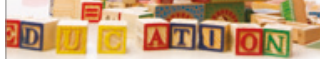
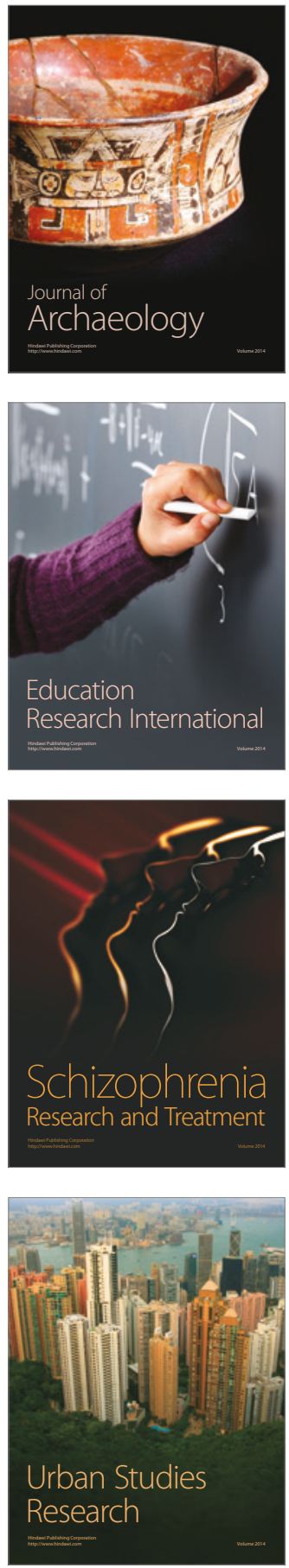